\title{
Vertebral osteomyelitis following manipulation of spondylitic necks - a possible risk
}

\author{
M Lewis BM, ${ }^{1 *}$ D Grundy FRCS ${ }^{2}$ \\ ${ }^{1}$ Senior House Officer, ${ }^{2}$ Consultant in Spinal Injuries, Duke of Cornwall Spinal Treatment \\ Centre, Odstock Hospital, Salisbury, Wilts, SP2 8BJ, England.
}

\begin{abstract}
Neurological complications of neck manipulation are well recognised but are usually due to acute vascular incidents. However, we describe two patients with cervical spondylosis, who developed staphylococcal osteomyelitis of the cervical spine with progressive tetraplegia, apparently following manipulation of the neck by a chiropractor. Although it is possible that the manipulation resulted in cervical spine trauma sufficient to cause local haemorrhage, the area becoming a nidus for infection, it is also conceivable that the patients underwent neck manipulation in an attempt to relieve pain due to an already existing osteomyelitis of the cervical spine, and the manipulation may have hastened the onset of spinal cord paralysis. Clearly, this could have occurred, as the average time between the onset of symptoms and diagnosis of vertebral osteomyelitis in most published series is about 2 months. Approximately $80 \%$ of cases of osteomyelitis occur in the 50-70 age group, a group in which cervical spondylosis is extremely common. It would seem that neck manipulation is particularly contraindicated in older patients with cervical spondylosis.
\end{abstract}

Keywords: vertebral osteomyelitis; neck manipulation; cervical spondylosis.

\section{Case reports}

\section{Case 1}

A fit 61 year old woman presented to her general practitioner with generalised neck ache. Muscle spasm was diagnosed after cervical spine radiographs were reported as normal. Four weeks later, due to persistence of neck pain, she consulted a chiropractor who, reassured by the normal radiograph report, manipulated her neck and applied megapulse twice weekly for 3 weeks.

Three days following her final treatment, she noticed weakness of the right arm, followed by the left arm, starting proximally and spreading distally. Within 7 days she was unable to lift either arm sufficiently to feed herself and became weak in both legs, being unable to walk unless supported by her husband. When seen, she gave no history of recent upper respiratory tract symptoms. She was apyrexial and non

*Correspondence: Woolston, Surgery, 66 Portsmouth Road, Woolston, Southampton SO2 9AL. toxic, and though her white blood count was normal her erythrocyte sedimentation rate was grossly raised $(130 \mathrm{~mm} /$ hour $)$. On examination, her left and right upper limbs were weak, with MRC grade 3 and 2 movements respectively. The power in the lower limbs was normal apart from MRC grade 4 hip flexion.

Review of the original radiographs showed mild cervical spondylosis but a repeat view now revealed destruction of the bodies of $\mathrm{C} 4$ and $\mathrm{C} 5$ with obliteration of the intervertebral disc space and a very large retropharyngeal swelling (Fig 1).

Biopsy of the body of $\mathrm{C} 4$ yielded pus, culture of which grew Staphylococcus aureus, sensitive to flucloxacillin. She completed 3 weeks of IV flucloxacillin followed by 3 weeks of oral therapy. Her neurological state improved rapidly and a month later she had regained normal power in the left, and MRC grade 4 movement in the right upper limb. Six months after the initial episode, radiographs showed bony consolidation and, after a further 6 months, neurological recovery was complete. 


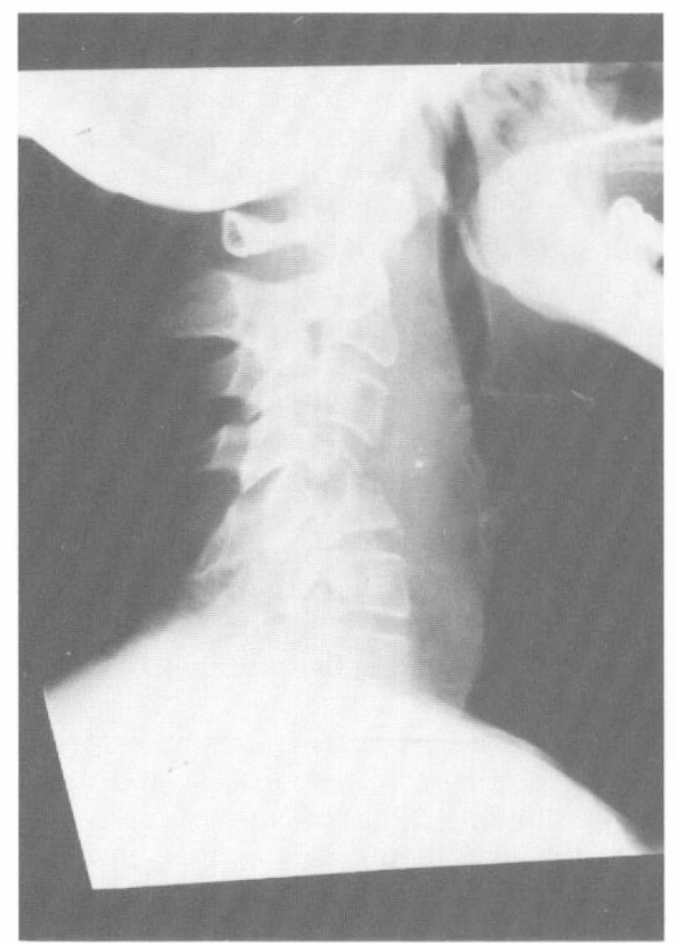

Figure 1 C4-5 osteomyelitis showing prominent retropharyngeal swelling.

\section{Case 2}

A 60 year old man with a life-long history of atopic eczema had a 2 year history of intermittent neck pain, attributed to cervical spondylosis, changes of which were present on radiographs. Two weeks following a chiropractic manipulation of his neck, he was admitted with progressive tetraplegia, developing over a few hours. All 4 limbs were hypertonic with MRC grade 1 power and a sensory level at $\mathrm{C} 4$. Radiographs now revealed erosion of the bodies of $\mathrm{C} 4$ and $\mathrm{C} 5$ with obliteration of the disc space (Fig 2).

Infected bone and disc material were resected and Staphylococcus aureus was cultured from the specimen. He was commenced on IV flucloxacillin and fusidic acid and his neck immobilised in a Halo vest.

Postoperatively, he presented with a central cord syndrome with flaccid arms and hypertonic legs. He regained MRC grade 3-4 power in the right upper limb and MRC grade 2-3 power in the left but had a complete spastic paralysis of the legs. He had patchy sensory loss to pinprick below C6 but normal posterior column function. One year after surgery, he had made

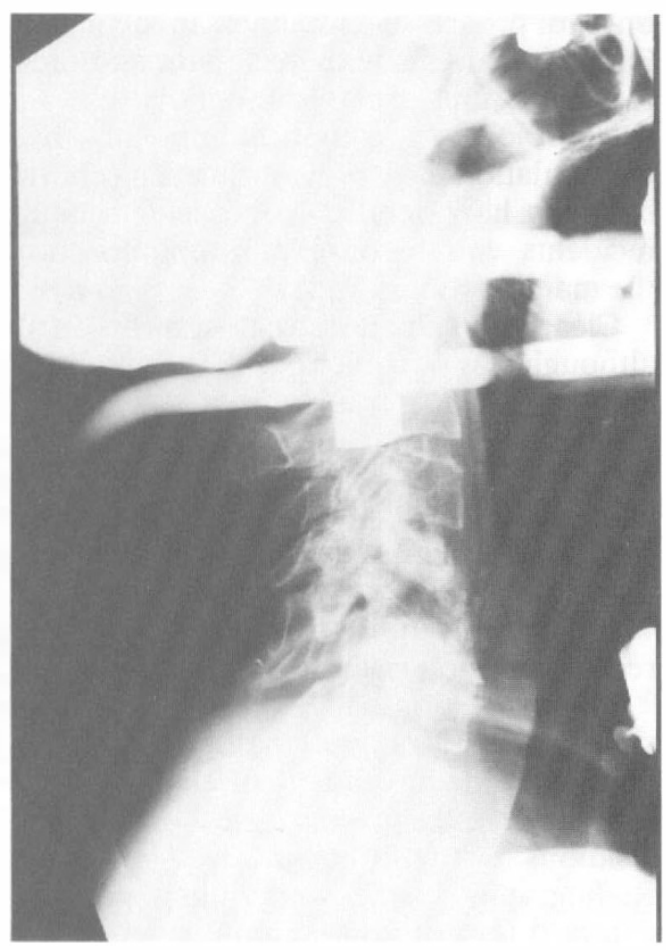

Figure 2 C4-5 osteomyelitis in a markedly spondylitic neck.

significant neurological recovery, particularly in his lower limbs, and was almost able to walk. Cervical spine films showed good regrowth of bone.

\section{Discussion}

The source of staphylococcal infection remains obscure in the first patient but it is possible that manipulation was sufficiently traumatic to cause a retropharyngeal haematoma, which subsequently became infected from the oro- or naso-pharynx before extending to involve the vertebrae. Another possibility is that of haematogenous spread from a distant focus.

In the second patient, with atopic eczema, the skin could have been the portal of entry for a staphylococcal infection. Neither patient reported a sore throat or upper respiratory symptoms that could have represented a source of local infection.

The only common history was that of cervical manipulation shortly before the 
onset of progressive weakness involving all 4 limbs, in patients with neck pain and radiological features of cervical spondylosis.

Neurological complications of neck manipulation have previously been reported but they have tended to be acute vascular incidents with the onset of symptoms during the manipulation. ${ }^{1}$

Clearly this did not occur in our patients, although it is possible that the manipulation was sufficiently traumatic to cause local haemorrhage, the area then becoming a nidus for blood-borne infection. We have been unable to trace a report of this occurring in the literature, except to note a report describing osteomyelitis in 2 patients, where the infection was superimposed on a recent vertebral fracture. ${ }^{2}$

However, another explanation is possible, when it is realised that the diagnosis of vertebral osteomyelitis is often delayed for over 2 months, ${ }^{3}$ the onset often being insidious with little malaise or fever. ${ }^{4}$ Radiographic changes may likewise not be detected for one to two months after onset of the infection. ${ }^{4}$
This then raises the question as to whether the neck pain, which prompted the patients to consult a chiropractor, was a symptom of undiagnosed cervical osteomyelitis, rather than cervical spondylosis. Eighty percent of patients with spinal cord paralysis due to vertebral osteomyelitis are in the 50 to 70 age group, ${ }^{5}$ a time when cervical spondylosis is extremely common. It would seem likely that neck manipulation of a patient with cervical vertebral osteomyelitis might precipitate or, at least, hasten signs of spinal cord paralysis.

Although the aetiology of the vertebral osteomyelitis in our two patients remains unclear, neck manipulation for pain would seem to be particularly contraindicated in older patients and in the presence of cervical spondylosis.

In the case of vertebral bony infection, urgent drainage of the abscess and appropriate administration of antibiotics is vital to give the patient the best chance of neurological recovery but, in spite of prompt treatment in both our patients, only one made a satisfactory recovery.

\section{References}

1 Smith RA, Estridge MN (1962) Neurologic complications of head and neck manipulations. JAMA 182: 528-31

2 Lowe J, Kaplan L, Liebergall M, Floman Y (1989) Serratia osteomyelitis causing neurological deterioration after spine fracture. J Bone Joint Surgery 71B: 256-258.

3 Liebergall M, Chaimsky G, Lowe J, Robin GC, Floman Y (1991) Pyogenic vertebral osteomyelitis with paralysis. Clin Orthop 269: 142-150.

4 Musher DM, Thorsteinsson SB, Minuth JN, Luchi RJ (1976) Vertebral osteomyelitis. Still a diagnostic pitfall. Arch Intern Med 138: 105-110.

5 Eismont FJ, Bohlman HH, Soni PL, Goldberg VM, Freehafer AA (1983) J Bone Joint Surgery 65A: 19-29. 